\title{
Experiences of physical complications and sequelae among living liver donors
}

\author{
Sun Ju Jeong, Han Na Kim \\ Department of Nursing, Suwon Women's University, Suwon, Korea
}

\begin{abstract}
Background: This study aimed to investigate the aspects of physical complications and sequelae of living liver donors in the context of the Korean culture. A deeper understanding of these experiences will provide basic data necessary for medical management programs for living liver donors and for those expecting to become living liver donors.

Methods: We used a descriptive ethnographic research method through in-depth interviews and participant observation. Data were collected from January 2016 to December 2017, till adequate quantity of data was obtained. Data were collected from 11 living liver donors using the "snow ball" method. In-depth interviews were conducted two to five times per participant, and the duration of each interview was 2 to 3 hours.

Results: The results were organized into one domain, three categories, and 12 subcategories. The domain of "physical sequelae remaining after donation" was derived from "experience of internal organ disorders," "long-lasting chronic pain," "decrease in immunity and increase in disease incidence." The experiences of "experience of internal organ disorder" were described as feelings of physical constraints similar to being disabled, $100 \%$ recovery to predonation status is not possible, markedly different stamina compared to that before donation, and fatigue, with increased difficulty in performing the activities of daily life.

Conclusions: Provision of appropriate medical care and continuous and systematic health care consultation before and after donation, and development of adequate support systems for donors are essential.
\end{abstract}

Keywords: Living donors; Donor's complication; Sequelae; Liver transplantation; Organ transplantation

\section{INTRODUCTION}

Living liver donor transplantation has improved significantly since 1979, when cyclosporin was introduced as a new immune inhibitor. Since then, demands among patients who require organ transplantation have been continually increasing [1]. The number of patients requiring transplantation has increased rapidly; however, organ donation from brain-dead donors has lacked to a serious

Received March 15, 2019

Revised May 10, 2019

Accepted May 16, 2019

Correspondence to: Han Na Kim

Department of Nursing, Suwon Women's University, 72 Onjeong-ro, Gwonseon-gu, Suwon 16632, Korea

Tel: +82-31-290-8234, Fax: +82-31-290-8208

E-mail: sunjuright@swc.ac.kr degree. Accordingly, the dependence on living liver transplantation in Korea remains dominant [2,3]. In the case of liver transplantation, in particular, one-fourth of living liver transplantation surgeries around the globe are performed in Korea [4].

Living liver transplantation describes the transplant of part of the organ from one living healthy individual to another. This is possible only when the removal of part of the organ causes no harm to the health of the donor. However, living donors often experience the physical burdens of organ transplantation and postoperative complications. Moreover, stable health is not necessarily guaranteed after donation $[4,5]$.

After the surgery, living liver donors (LLDs) may experience bile leak, bleeding, portal vein thrombosis in the liver, wound infection, seroma, atelectasis, pleural effusion

This is an Open Access article distributed under the terms of the Creative Commons Attribution Non-Commercial License (http://creativecommons.org/ licenses/by-nc/4.0/) which permits unrestricted non-commercial use, distribution, and reproduction in any medium, provided the original work is properly cited. 
fluid, ileus, cicatrix, hernia, ascites between organs, enteroplegia, and/or other serious complications, some of which may even lead to death [4-8]. Furthermore, it has been reported that after leaving the hospital, LLDs experience complications and sequelae, including digestive disorders, stomachache, fatigue, headache, loss of appetite, wound, dyschezia, and dysuria; some of these complications may be serious enough to require hospitalization and/or pharmacological therapy thereafter $[4,8,9]$.

In addition, LLDs experience feelings of loss due to the removal of a part of their bodies after surgery, serious pain, and aesthetic damage due to scarring from the surgical incision. It has also been reported that they encounter physical, psychological, social, and economic challenges after surgery due to the limited range of physical activity and changes in health condition, as well as restrictions to social activities [4,9-12].

Despite such needs, however, previous studies investigating living liver transplantation have focused on technical issues, post-surgery complications and quality of life $[1,2,4]$, while there have been limitations in the understanding of various physical complications and sequelae among donors. In particular, there have been only a few in-depth qualitative studies investigating the physical experiences of LLDs [9,13], and very few related topics have been addressed in other studies, both domestically and abroad.

In Korean culture, living liver transplantation may lead to various problems for the donor, in addition to the medical act of organ removal because pa art of an organ is taken from a living person to treat a disease- not in oneselfbut in another. In Korea, living liver transplantation is projected to increase as the only recourse to save the lives of terminal patients with organ failure until organ donation from brain dead donors becomes more common. Thus, it is necessary to explore and understand the unique experiences of LLDs.

Accordingly, this study aimed to thoroughly examine the physical complications and sequelae that LLDs experience in the sociocultural context of Korea. In addition, basic information about how to manage potential or actual LLDs, develop education programs, and arrange support systems is suggested based on a deeper understanding of their experiences.

\section{METHODS}

\section{Research Design}

This qualitative study applied an ethnographic research method to examine specific physical complications and sequelae that LLDs experience in the sociocultural context of Korea, and was based on in-depth interviews and participation observation, which are field research methods.

\section{Research Participants (Informants)}

Participants in the present study were LLDs who donated part of their organ at least 1 year previously. All participants understood the objective of the study and agreed to participate. LLDs whose donation was performed at least 1 year previously were selected because they were not fully recovered, even 1 year after donation, and/or in whom physical and mental difficulties persisted even after years $[4,10,13]$. Data collection and analysis were conducted among 11 LLDs to determine the timing of theoretical saturation.

Of the donors, six were men and five were women. At the time of liver donation, one donor was a teenager, three were in their 20s, four in their 30s, two in their 40s, and one in his 50s; all donors donated the right lobe of their liver. The relationship between the donors and recipients was as follows: in seven cases, the donors were children donating to their parents; in one case, a wife donated to her husband; one donor donated to an aunt; in one case, the son-in-law donated to his farther-in-law; and one donor donated to an acquaintance. The period since donation ranged from 1.6 to 12 years.

\section{Data Collection}

Data collection was initiated after approval from the National Bioethics Committee (IRB No. HIY-14-095-4). To ensure voluntary and ethical participation of the study subjects, each subject was informed of the research objective, methods, and interview content. The in-depth interviews were recorded to prevent omitting of details and to ensure accuracy. After the participants were informed that all the collected materials, including recordings, 
would be used only for academic research, that the analysis and interpretation of the results throughout the study would be conducted anonymously, and that they could withdraw from the study at any time, they signed the agreement for participation in the study.

As the lead investigator of this study is also an LLD (donated in 2008), efforts were made to maintain objectivity throughout the process by avoiding prejudices and conducting in-depth interviews with individual participants instead of relying on the experiences of LLDs described in previous studies. The literature on LLD experiences was reviewed to enhance theoretical sensitivity, and the questions were so drafted to derive new findings and collect data.

Participants were selected from among liver donors introduced by the Korean living organ donor association (a meeting of liver donors and living organ donors), and data were collected from those who came into direct contact and participated in the research.

Data collection was conducted for approximately 2 years from January 2016 to December 2017. The in-depth interviews and participant observation continued to data accumulation until finally reaching the point of saturation. Two to five interviews were conducted with each participant, with each lasting approximately 2 to 3 hours. The interviews were conducted at venues where the participant or the researcher would not be hindered, including the participant's home, office, or a cafe where they would feel most comfortable. Informal and open questions were posed. For example, questions such as "How is your daily life lately?" and "How was your daily life after donation?" were asked first; the participants' answers then led to other questions.

During the interviews, the participants' nonverbal expressions, such as facial expressions and tone of voice, were carefully observed, and notes were taken on site. Recordings of in-depth interviews were transcribed by the researcher immediately after the interviews. The recordings were replayed repeatedly to verify meaning and avoid missing or changing any of the statements of the researcher or participants, the contents were transcribed verbatim. After the interview contents were verified, notes were taken regarding aspects that needed further specifi- cation, and then the meaning and content were reconfirmed by the participants in following interviews, whether online or via e-mail, or on the telephone.

\section{Data Analysis}

Data analysis was conducted as an ongoing process simultaneous with data collection. Based on the process of analyzing domains, categories, subcategories, and themes was conducted as suggested by Spradley [14] to determine and interpret cultural themes from living donors' personal experiences. To understand the experiences of LLDs, the repeated and regressive process of asking, listening, exploring, comparing, contrasting, composing, checking, and evaluation continued.

Based on the analyzed experiences of LLDs, consultation and confirmation were conducted with two professors experienced with the ethnographic research method and one physician experienced with transplantation of a living donor's organ. In the denominating process, one professor specialized in qualitative research and one who majored in education studies joined the discussion. Finally, one professor of nursing studies was consulted for evaluation. In addition, a validation process was conducted among three major informants to verify semantic congruence of the analysis results.

\section{RESULTS}

The experiences of physical complications and sequelae among LLDs in the sociocultural context of Korea were classified into one domain, three categories, and 12 subcategories (Table 1). In the domain of physical sequelae left after donation, experience(s) of internal organ disorder, long-lasting chronic pain, and decrease in immunity and increase in disease incidence, were derived.

\section{Experience of Internal Organ Disorder}

LLDs experienced the following internal physical disorders: "feeling physical constraints similar to those of the disabled"; "100\% recovery to pre-donation status is not possible"; "markedly different stamina compared with before donation"; and "fatigue, with increased difficulties in daily life." 
Table 1. Physical complications and sequelae experienced by living liver donors in Korea

\begin{tabular}{|c|c|c|}
\hline Domain & Category & Subcategory \\
\hline \multirow[t]{7}{*}{$\begin{array}{l}\text { Physical sequelae } \\
\text { after donation }\end{array}$} & Experience of internal organ disorders & $\begin{array}{l}\text { Feeling physical constraints similar to being disabled } \\
100 \% \text { Recovery to predonation status is not possible } \\
\text { Markedly different stamina from that before donation } \\
\text { Fatigue with increased difficulty in performing daily life activities }\end{array}$ \\
\hline & Long-lasting chronic pain & $\begin{array}{l}\text { Pain in the abdomen, shoulders, back, and calf } \\
\text { Hand and foot numbness, and paralysis symptoms } \\
\text { Discomfort with surgical scarring in the operative area }\end{array}$ \\
\hline & $\begin{array}{l}\text { Decrease in immunity and increase } \\
\text { in disease incidence }\end{array}$ & $\begin{array}{l}\text { Increased incidence of inflammatory diseases, such as enteritis, cystitis, } \\
\text { dermatitis, and eye inflammation }\end{array}$ \\
\hline & & Liver enzyme level elevation and fatty liver and hyperlipidemia \\
\hline & & Indigestion and changes in bowel movements/habits \\
\hline & & High blood glucose levels and diabetes \\
\hline & & $\begin{array}{l}\text { Increases in the incidence of diseases of the female reproductive system, such } \\
\text { as menstrual irregularity, menstrual pain, breast masses, uterine leiomyoma }\end{array}$ \\
\hline
\end{tabular}

\section{Feeling physical constraints similar to those} experienced by the disabled

LLDs felt as if their physical ability resembled that of the disabled, while experiencing changes in life and incomplete physical recovery after liver donation. They reported that liver resection (i.e., donation) would not be recognized as a disability because it was not visible, while donation of an arm or leg would be easily noticed and recognizable. The donor responded that he/she experienced sequelae after donation.

I feel sequelae after donation. Major sequelae are internal disability, functional failure, and feelings of incompetence. These are deteriorating as time passes (LLD 1-11). If I cut off my arm and donated it to someone, people would easily notice it. However, it is not the case for donors like me. Surgeries we went through were for parts unseen, and people could hardly notice what have been done inside of us $\cdots$ (LLD 6). I have no right lobe. I hear that it grows, but actually, it is the left lobe that grows. I feel disability in various aspects including physical strength. It is unseen. You might look normal when seen outside, but the organ is no longer complete $\cdots$ (LLD 8). I was not fully recovered. I did not know before I actually underwent the operation. It was much more painful than expected. No complete recovery, and even there were sequelae. I was completely healthy, but now I have this disability that remains forever (LLD 1).

\section{It will be impossible to $100 \%$ fully recover to the state before donation}

All LLDs believed that their physical condition was not as good compared to that before donation. Even years after donation, the donors felt that they were not fully recovered and that it would be absolutely impossible to recover to the condition before donation.

Even wounds leave traces. It is difficult to fully recover after organ resection. Many things changed after donation. I feel different. I would never be recovered to the state before donation (LLD 1-11). It has been 10 years since I donated. As I feel that my body changed over time, and I feel the clear difference before and after donation. My physical strength is also not as good as before (LLD 8). I feel easily tired even while performing my usual daily activities. It is difficult to explain the difference between being arduous and being tired. My mind is awake but my body is so tired out that I feel like I could do nothing in daily life. It is only $50 \%$ as good as before donation (LLD 6). I did everything I could, exercising, riding a bike, etc. No matter what I might do, I could not go back. Thereafter, I thought that I just had to admit it and live this way until the end of my life (LLD 1).

\section{Markedly different stamina compared with before donation}

All LLDs experienced a decline in physical strength com- 
pared to that before donation; in some cases, this was described as significant and serious. Even many years after donation, the physical strength deteriorated to the point where it was difficult to focus on work for a 2-hour period.

I feel I am easily tired out after donation (LLD 1-11). Almost all donors would feel such feelings of physical strength loss. It might look seemingly okay, but the physical strength is certainly not as good as before (LLD 5). Before donation, I need very little sleep while working hard even for a week. After donation, well, I do not know about other donors, but I go out and feel tired out only within 2 hours. 2 hours! I feel tired, exhausted with facial muscles hardened, and I am totally burned out. I feel exhausted even if there is delicious food right in front of me. I know that I have to feed myself somehow, but I even have no strength to eat. I have no physical strength enough to focus on something for 2 hours (LLD 6).

\section{Fatigue, with increased difficulties in daily life}

All LLDs complained that they felt tired easily and experienced chronic fatigue after donation. They reported that their fatigue became so serious that it significantly affected their daily and social lives. Consequently, they easily experienced negative feelings and became irritable due to their deteriorating quality of life.

After donation, I feel too much tired and hard. I feel easily exhausted (LLD 1-11). Fatigue is so serious that even right after I wake up, I feel as if I was beaten up while sleeping. I feel under the weather and sluggish and as if I was beaten up when waking up in the morning. Such feelings were quite serious for 6 months to 1 year. I realized that when a person lost his or her health, the quality of life would decline sharply $\cdots$ (LLD 6). After donation, I would be easily tired and so sleep just like a newborn baby. My family might think that this is my normal sleeping pattern, but actually I feel too much tired out (LLD 7).

\section{Long-Lasting Chronic Pain}

LLDs experienced long-lasting chronic pain including "chronic pain in the abdomen, shoulder, back, and calf," "hand/foot numbness and paralysis," and "discomfort for the scars after surgery," among others.

\section{Chronic pain in the abdomen, shoulder, back, and calf}

LLDs whose right upper liver quadrant was removed complained about pain at the right shoulder, right limb, right side and, particularly, the right upper quadrant. Some liver donors also experienced squeezing pain at the calf.

Sometimes I feel uncomfortable stiff at the right shoulder. I feel pricking around the liver. I feel pain though it is normal when examined (LLD 8). I feel pain at the right abdominal area and side. The pain and discomfort last for a long time (LLD 3). Stomachache never stops. I feel stomachache which annoys me all the time (LLD 9). Even now, I feel as if something like a disinfectant or lox is burning all over my body. I feel like muriatic acid is pouring over my stomach... Under the leg, I feel as if someone is squeezing the muscles. Muscular pain over the calf also remains. My leg is twisted while I sleep $\cdots$ (LLD 6).

\section{Hand/foot numbness and paralysis}

Some LLDs reported hand/foot numbness and paralysis that they never experienced before donation. Their numbness and paralysis were so serious that they even needed medication(s).

I had these feelings of hand/foot numbness that I had never had before donation (LLD 1, 6, 8, 10). I hear that this symptom results of blood circulation disorder. The numbness often leads to serious shooting pain. I am prescribed medicines for hand/foot numbness, paralysis, and peripheral nerve disorder (LLD 9).

\section{Discomfort from surgical scars}

Some LLDs experienced wound opening and infection. The serious pain continued after surgery. Among many of the donors, discomfort from parts of the incision continued for a long time. Pain resembling stabbing with a scalpel persisted, even after 10 years. 
I feel pulled over the abdominal area, and I could not walk properly (LLD 1). I feel as if someone stabs with a scalpel even while I am doing nothing. The pain! It remains for a while. It returns suddenly! I feel like I am going crazy $\cdots$ (LLD 9). I even could not wear a brassiere. Since it comes over here and tightens this area, I sometimes have to release it. When it is twisted over here! I feel as if it pecks me. It has passed 10 years since I donated, but the discomfort at the spot of incision still remains (LLD 10). The discomfort and pain at the spot of incision still remain ( $\mathrm{LLD} 3,5$, and 7).

\section{Decrease in Immunity and Increase in Disease Incidence}

LLDs experienced decrease in immunity and increase in disease incidence including, the following symptoms: increase of inflammatory diseases such as enteritis, cystitis, dermatitis, eye inflammation; liver enzyme level elevation; fatty liver and hyperlipidemia; indigestion and changes in bowel movements; high blood sugar levels and diabetes; menstrual irregularity, menstrual pain increase, breast mass, fibroid; and increases in other diseases of the female genitalia.

Increases in inflammatory diseases including enteritis, cystitis, dermatitis, and eye inflammation

All LLDs were healthy before donation. After donation, however, increase in the incidence of diseases, including frequent infections and colds, enteritis with an unknown cause, cystitis, skin irritation, hives, pruritus and eye irritation, among others, were reported. Decrease in immunity was another symptom. This was not merely a subjective recognition of infection, but actual increase in inflammation indices in laboratory tests. Some donors even experienced leishmaniasis due to long-term and frequent inflammation, while others experienced shingles.

After the surgery, I experience inflammation and infection often. My immune system is also not as good as before (LLD 3). Certainly, my immune system is vulnerable. Inflammation is frequent, and the cold, once I caught, lasts longer than before (LLD 2). Liver function test results show that it is normal, but the symptoms come along with en- teritis seasonally. I have skin irritations, swellings, and hordeolum that I had never had before. Such irritations occur frequently (LLD 7). I received 2-week dosage of antibiotic agents since the inflammation index was too high. I took injections as well as antibiotic drips. I also have cystitis. In general, inflammation indexes have increased and kala azar has deteriorated excessively as my immunity has dropped (LLD 6). I feel itchy on my skin. I also have indigestion and hives that I had never had before (LLD 8). Inflammation indexes increased. I has taken hospital treatment every year. I had shingles too, which was terribly hard (LLD 5 and 9).

\section{Liver enzyme level elevation, fatty liver, and hyperlipidemia}

Even 1 year after donation, the serum glutamic-oxaloacetic transaminase/serum glutamate-pyruvate transaminase liver somatic index was as high as 100 in some LLDs. Fatty liver syndrome deteriorated to the point of hepatocirrhosis in some cases. I was diagnosed with hyperlipidemia and has taken medications for it. Before donation, I was healthy with no liver functional problems or hyperlipidemia in tests. After donation, the one and only organ has functional disorder, making me worry about my health.

The liver somatic index is as high as 100. When I had the examination right before the surgery, I had no fatty liver syndrome. After the surgery, my body condition went terribly bad. When going to the hospital, doctors told me that the fatty liver syndrome was serious, prescribing medicines for hyperlipidemia as well. The liver has recovered to the original size, but the quality has deteriorated to the point of hepatocirrhosis (LLD 6).

Indigestion and changes in bowel movements/habits Most LLDs reported functional gastrointestinal disorders. They also complained of changes in bowel movements including indigestion, diarrhea, and constipation after meat and fat intake, as well cramps and discomfort in the stomach. LLDs emphasized that indigestion was the most significant change before and after surgery. 
The biggest change after donation is indigestion (LLD 111). The most serious is that I cannot digest certain foods (LLD 5). I cannot eat much. I cannot digest most meats such as chicken and pork. I eat fish but only a small amount (LLD 1). I often have digestion problems and bloated stomach. I would like and eat a lot of meats, but now I cannot digest once I eat them (LLD 7). Pork belly! Chicken! I would eat such things often at night, but after donation, I cannot because of digestion problems. I eat vegetables only. Things with no fat. Stomach convulsion makes me roll. Volvulus is really painful (LLD 2, 8, and 10).

\section{High blood glucose levels and diabetes}

Some LLDs with no symptoms of diabetes before donation experienced diabetes and high blood glucose levels after donation. In the case of $1 \mathrm{LLD}$, the blood glucose index was as high as $300 \mathrm{mg} / \mathrm{dL} 8$ years after donation. Presently, this donor is still taking medicines for diabetes and managing blood glucose levels. Some donors experienced impaired glucose tolerance, which is a symptom of high postprandial glucose, and required intermittent medicine intake, dietary modification, and regular exercise.

The blood sugar level is too high after meals. It is as high as 300. I have diabetes now. Now it has passed 8 years after donation, and the blood sugar level is as high as 300. I take prescribed medicines for diabetes (LLD 9). The fasting blood sugar level is also as high as 100 to 120 . The postprandial sugar level is high, sometimes as high as 200 depending on the food I eat. After donation, the blood sugar level is so instable that I have to regulate food I eat (LLD 1, 6, 8, and 10).

Menstrual irregularity, menstrual pain increase, breast mass, fibroids, and increases in other diseases of the female genitalia

Some female LLDs experienced amenorrhea for 2 to 3 months immediately after donation, irregular menstrual cycles after donation, and other irregular menstruation symptoms, such as menstrual pain. Among some LLDs, multiple masses grew in their breasts, in addition to fibroids and cysts. Some donors required resection of uterine or ovarian polyps 1 month after the surgery. Immediately after surgery, some donors also experienced galactorrhea. They also experienced dyslochia similar to when they were pregnant and gave birth.

Irregular menstruation symptoms were quite serious for 2 to 3 months after liver donation. The period came even twice a month, and sometimes there was no menstruation at all. It was quite irregular after donation probably the surgery was physically quite hard (LLD 4, 6, and 10). After the liver surgery, I experienced lactation just as I breast-fed a newborn baby. I also experienced dyslochia just like when I bread-fed my baby. It had not been this much before, but as a menstruation starts, the pain is so much that it is difficult to get up.. (LLD 9). I am not married yet, but I had to undergo an operation 1 month after liver donation due to the uterine polyp, ovary polyp, and fibroid symptoms. Before the surgery, I heard that there would be no problem, but I had to undergo one big operation after another thereafter (LLD 4).

\section{DISCUSSION}

This qualitative study applied an ethnographic research method to examine specific physical complications and sequelae that LLDs experience in the sociocultural context of Korea. The analysis revealed that after liver donation, LLDs experienced physical sequelae including internal organ disorder, long-term pain, and decreased immunity and increased disease incidence, among others. After donation, LLDs experienced changes in life, feeling physical constraints similar to those of the disabled, and incomplete physical recovery. The extent of feeling physical constraints similar to those of the disabled was particularly significant. They felt that their physical conditions were not as good as before donation and complained of markedly altered stamina from before donation. Even after many years, they quite often experienced fatigue, making their daily activities challenging, if not exhausting.

According to many previous domestic and foreign studies, LLDs experience pain more serious than expected, markedly different stamina from before donation, frequent chronic fatigue, indigestion, gastrointestinal disorders, 
such as stomachache, sleep disorders due to physical discomfort, discomfort from the area of the incision, and inflammation due to decreases in immunity $[4,10,12,15]$. These findings are consistent with the physical sequelae that subjects in this study experienced after donation.

In addition, subjects experienced frequent fatigue, failed to recover to their predonation state, and lost physical strength with feelings physical constraints similar to those of the disabled, even years after the surgery [15]. In particular, all LLDs experienced significant changes in bowel habits, including diarrhea and constipation, gastrointestinal disorders, such as indigestion, and stomach cramps and discomfort. They also complained about abdominal pain, and pain in the right shoulder, sides, and calf. One report from the Japanese Liver Transplantation Research Institute [10] also reported that, while doctors did not recognize them, a significant number of liver donors experienced subjective difficulties such as abdominal discomfort, gastrointestinal disorders, and stomachache. Such findings are consistent with the results of this study regarding physical sequelae. It is necessary, therefore, for medical teams to devote special attention to symptoms that donors complain about and to provide sufficient methods for medical management.

Because LLDs are physically healthy adults, it is generally assumed that they will recover to an almost normal condition with no major problems. However, findings of this study indicate that there were significant differences before and after the surgery among LLDs, particularly regarding physical sequelae.

LLDs experienced slow physical recovery and decreases in immunity, resulting in frequent inflammatory disease of the intestines, respiratory diseases, irritations of the bladder, skin and eyes, and hives. Furthermore, inflammation indexes increased significantly, even involving leishmaniasis. Some LLDs experienced organ function disorders, involving increases in liver index, fatty liver syndrome, hyperlipidemia, and hepatocirrhosis. Some donors experienced high blood glucose levels and diabetes.

Even after leaving the hospital, some donors often had to visit the hospital and drug stores to remedy indigestion, stomachache, fatigue, loss of appetite, wounds, dyschezia, and dysuria. Such experiences affected recovery to normal daily life $[8,10]$. Brown et al. [16] reported that while the death rate of LLDs was low, the frequency of complications among them was relatively high. After surgery, LLDs may experience bile leak, bleeding, portal vein thrombosis, wound infection, seroma, atelectasis, pleural effusion fluid, ileus, cicatrix, hernia, ascites between organs, enteroplegia, and/or other serious complications, some even causing death $[7,8]$. Moreover, it has been reported that they experience problems such as indigestion, stomachache, fatigue, loss of appetite, wounds, dyschezia, and dysuria $[7,8]$.

Complications after liver donation are problems that occur not only in liver donors. According to previous studies investigating complications among kidney transplant donors, complications including kidney functional disorders, high blood pressure, and albuminuria occur, causing serious safety risks $[17,18]$, even to the point of death. In addition, many donors experience physical problems such as indigestion, stomachache, fatigue, cold, cardiac inflammation, urolithiasis, albuminuria, headache, and kidney inflammation, which prompted them to visit the hospital or drug stores after donation [2]. Although LLDs experience various physical complications and sequelae, in reality, no systematic health management is practiced among this patient population $[4,15]$.

It is necessary, therefore for the government to take proper measures such as funding for regular health examination, medical treatment, and sequelae management for individuals who have donated part of their organs to save lives. Particularly, such physical problems cause psychological and mental symptoms and negatively affect their return to society $[4,15]$. Keen attention must be devoted to symptoms that liver and kidney donors complain about. In addition, surveys investigating the actual conditions of physical changes as well as disease histories need to be conducted, with a proper health management system established for them.

The female LLDs who participated in this study experienced breast- and genitalia-related diseases that have not been reported in previous studies. Donors also experienced amenorrhea, menstrual irregularity, increase in menstrual pain, breast masses, hyperlactation, fibroids and cysts, among others. Some donors required resection of 
the uterine and/or ovarian polyps 1 month after the surgery. It is necessary to perform a detailed evaluation of each donor's health condition, including physical examination with respect to the organ to be donated, sex, age, family disease history, and other organ conditions and pregnancy, among others. In addition, tests must be conducted to assess the incidence of various cancers, such as cervical, breast, prostate, and colorectal cancers. When a donor is selected, the doctors must provide the donor with sufficient information about his/her health condition based on the detailed evaluation results.

Finally, living organ donors experience significant physical limitations to their daily and social lives for a long time after donation. Thus, they may be included in the concept of the "disabled" or "disability" specified in Article 2 of the Welfare of Persons with Disabilities Act [19]. The concept of disability or the disabled is not limited to permanent conditions but may include mental or physical limitations to daily and social lives for a long period of time. Thus, it is necessary to revise the relevant laws so that donors can be registered as disabled persons in accordance with Article 32 of the present registration of the Disabled Persons Act [10,19]. It is also necessary to conduct a wider societal discussion regarding the necessity of expert medical review for living donors to be able to be registered as disabled persons.

This study evaluated the physical complications and sequelae experienced by the LLDs after liver donation. After donation, the donors experienced significant internal organ disorders similar to those experiences by the disabled. They also experienced long-term pain and/or discomfort in the abdomen and at the surgical site, as well as decreased immunity, resulting in increased incidence of other diseases. Therefore, it is essential to make efforts and draft policies at the local and national level for the health and welfare of the living donors, as well as to preserve their rights. It is necessary to establish policies for living organ donation and to conduct thorough investigations of actual conditions for institutional improvement. In addition, mid/long-term plans, revisions of the rules and policies related to organ/liver donation legally, and institutional arrangements must be established as soon as possible to support living organ donors.
As this study involved only $11 \mathrm{LDS}$, there are limitations to generalizing the findings. However, our results remain significant in that they provide a basis for the appropriate management and development of an education program for individuals who are, or potentially could be LLDs; our findings may also aid in drafting plans to support this patient population.

\section{CONFLICT OF INTEREST}

No potential conflict of interest relevant to this article was reported.

\section{ACKNOWLEDGMENTS}

This study was conducted with support for research projects at Suwon Women's University in 2018 (2018-2017).

\section{ORCID}

Sun Ju Jeong https://orcid.org/0000-0002-2033-4935

Han Na Kim https://orcid.org/0000-0002-7370-1815

\section{REFERENCES}

1. Hwang S, Moon DB, Lee SK. Current status and perspectives of living donor liver transplantation. J Korean Med Assoc 2008;51:700-7.

2. Kim MH, Kwon OJ, Kang JM. The quality of life for living donors after kidney transplantation. J Korean Soc Transplant 2012;26:15-22.

3. Korean Network for Organ Sharing (KONOS). 2017 KONOS annual report [Internet]. Seoul: KONOS; 2018 [cited 2019 Jan 5]. Available from: https://www.konos.go.kr.

4. Korea National Institute for Bioethics Policy; Korean Network for Organ Sharing (KONOS). A study on the present state and improvement plan of discrimination and disadvantage for living organ donor. Seoul, KR: KONOS; 2012.

5. Helal I, Abdallah TB, Ounissi M, Tahar G, Cherif M, Boubaker $\mathrm{K}$, et al. Short- and long-term outcomes of kidney donors: a report from Tunisia. Saudi J Kidney Dis Transpl 2012; 23:853-9.

6. Ghobrial RM, Freise CE, Trotter JF, Tong L, Ojo AO, Fair $\mathrm{JH}$, et al. Donor morbidity after living donation for liver transplantation. Gastroenterology 2008;135:468-76.

7. Trotter JF, Talamantes M, McClure M, Wachs M, Bak T, 
Trouillot T, et al. Right hepatic lobe donation for living donor liver transplantation: impact on donor quality of life. Liver Transpl 2001;7:485-93.

8. Yoo JY, Yi NJ, Suh KS, Kwon JH, Choi SH, Lee KU. Donor quality of life in living donor liver transplantation. J Korean Soc Transplant 2004;18:73-80.

9. Jeong SJ, Yoo EK. The psychological and mental experiences of living liver donors in south Korea. Contemp Eng Sci 2014;7:1197-205.

10. Japanese Liver Transplantation Society (JLTS) \& Donors Research Committee. Research report for living-related liver transplantation. Osaka, JP: JLTS; 2005.

11. Euro Living Donor (EULID). Euro living donor project [Internet]. EULID; 2009 [cited 2019 Jan 5]. Available from: http://www.eulivingdonor.eu.

12. Manyalich M, Ricart A, Martínez I, Balleste C, Paredes D, Vilardell J, et al. EULID project: European living donation and public health. Transplant Proc 2009;41:2021-4.

13. Kang da HS, Yang J. Adaptation experience of living kidney donors after donation. J Korean Acad Nurs 2016;46:271-82.

14. Yoo EK. Ethnography and ethnographic research method. 1st ed. Seoul, KR: Soomoonsa; 2005.

15. Jeong SJ. Experience of living liver donor. Bioethics Forum 2012;1:208-15.

16. Brown RS Jr, Russo MW, Lai M, Shiffman ML, Richardson $\mathrm{MC}$, Everhart JE, et al. A survey of liver transplantation from living adult donors in the United States. N Engl J Med 2003;348:818-25.

17. Ibrahim HN, Foley R, Tan L, Rogers T, Bailey RF, Guo H, et al. Long-term consequences of kidney donation. $\mathrm{N}$ Engl J Med 2009;360:459-69.

18. Chang HK, Ju MK, Ahn HJ, Kim HJ, Jeon KO, Kim MS, et al. Long-term change of renal function after donor nephrectomy for kidney transplantation. J Korean Soc Transplant 2007;21:75-80.

19. Jwa SJ. Legal review of the recognition of disability by living liver donor. In: Korea Living Organ Donor Association \& The Human Rights Forum of Persons with Disabilities in Korea, eds. Discussion on ways to improve the system for living organ donors. Proceedings of 2014 Policy and Alternative Forum 3rd discussion forum; 2014 Nov 26; Seoul, Korea. p. 77-84. 